\title{
Prognosis of Hepatocellular Carcinoma after Liver Transplantation: Comparative Analysis with Partial Hepatectomy
}

Kyuho Lee $\cdot$ Kyoung-Bun Lee Nam-Joon $\mathrm{Yi}^{1} \cdot$ Kyung-Suk Suh ${ }^{1}$ Ja-June Jang

Departments of Pathology and 'Surgery, Seoul National University College of Medicine, Seoul, Korea

Received: September 19, 2016

Revised: October 4, 2016

Accepted: October 13, 2016

\section{Corresponding Author}

Kyoung-Bun Lee, MD

Department of Pathology, Seoul National University Hospital, 101 Daehak-ro, Jongno-gu, Seoul 03080, Korea

Tel: +82-2-2072-2968

Fax: $+82-2-743-5530$

E-mail:kblee@snuh.org

\begin{abstract}
Background: Liver transplantation (LT) is the treatment of choice for hepatocellular carcinoma (HCC). The aim of this study was to investigate the recurrence rate of HCC after LT and prognostic factors for recurrence by comparing LT with non-transplanted resection. Methods: The participants were 338 patients who underwent LT between 1996 and 2012 at Seoul National University Hospital (LT group) and 520 HCC patients who underwent partial hepatectomy between 1995 and 2006 (control group, non-LT group). Results: In the LT group, 68 of 338 patients (19.8\%) showed relapse, and the recurrence rate was lower than that in the non-LT group $(64.9 \%$, $357 / 520, p<.001$ ). Stratification analysis by American Joint Committee on Cancer (AJCC) stage showed that the stage I-II LT group had a lower recurrence rate than the non-LT group. Univariate comparative analysis demonstrated that multiplicity of tumor, tumor size, gross type, Edmondson-Steiner (ES) nuclear grade, extent of tumor, angioinvasion, AJCC stage, Milan criteria, University of California at San Francisco criteria on explant pathology (all $p<.001)$, positive expression of cytokeratin $19(p=.002)$, and preoperative $\alpha$-fetoprotein (AFP) $(p<.001)$ were predictors of tumor recurrence. In multivariate analysis, LT, preoperative AFP, multiplicity of tumor, extent of tumor, size of tumor, and ES nuclear grade were independent prognostic factors. Conclusions: LT might have a protective effect against the late recurrence of stage I-II HCC compared to non-LT, and the prognostic factors for recurrence were similar to previously well-known prognostic factors for HCC.
\end{abstract}

Key Words: Liver transplantation; Hepatocellular carcinoma; Recurrence; Prognosis
Hepatocellular carcinoma (HCC) is the fifth most common malignant tumor in the world. ${ }^{1}$ The treatments for HCC vary depending on the stage of the primary tumors and the patient's hepatic function. Resection is the treatment of choice for localized tumors, which are generally early-stage tumors, and liver transplantation (LT) is frequently selected as an alternative, potentially curative treatment for HCC.

After the Milan criteria for deceased donor LT for HCC were introduced in 1996, LT became an alternative treatment choice for early-stage or small HCC to achieve good disease-free survival (DFS). ${ }^{2}$ However, as living donor LT has rapidly increased due to the shortage of deceased donors in Eastern countries including Japan and Korea, the development of selection criteria of LT for HCC has become a dominant issue in this field that must include consideration of the balance between the benefit to the recipient and the risk to the liver donor. LT seems to result in a good prognosis for patients with early-stage HCC who are within the Milan criteria. The Milan criteria incorporate the size of the tumor, the number of tumors, and the presence of vascular invasion; patients eligible for LT are those with a single tumor $5 \mathrm{~cm}$ or smaller in size or 2-3 tumors each $3 \mathrm{~cm}$ or smaller in size, without macroscopic vascular invasion or extrahepatic spread according to preoperative radiologic findings. A low incidence of recurrence is expected among this group of patients. ${ }^{2}$ However, there is still a controversy about the cutoff points of tumor size and number of nodules. ${ }^{3,4}$ Accordingly, Yao et al. re-evaluated the upper limits and effects of tumor number and size in relation to DFS and proposed new criteria. The University of California at San Francisco (UCSF) criteria include the presence of a tumor $6.5 \mathrm{~cm}$ or smaller in diameter with a single nodule or no more than three tumor nodules, none exceeding $4.5 \mathrm{~cm}$ with a total diameter less than $8 \mathrm{~cm}$, and no vascular invasion according to pathologic evaluation. ${ }^{5}$ However, some patients who do not fulfill the Milan or UCSF criteria experienced a prolonged survival time, contrary to expectations. ${ }^{6,7}$ Lee et al. ${ }^{8}$ therefore suggested another set of criteria different from the previous measures and based on pathology. Their criteria are a largest tumor diameter of $5 \mathrm{~cm}$ or less, six or fewer tumors, and no gross vascular invasion. 
These criteria are valuable and have a prognostic power similar to that of the Milan and UCSF criteria. ${ }^{8}$ In addition to clinical and pathological prognostic factors, immunohistochemical factors are significant; cytokeratin 19 (CK19) is a representative factor. $^{\text {? }}$

According to a guideline for the management of hepatocellular carcinoma in Korea, deceased donor LT is the first choice of treatment for HCC within the Milan criteria (single nodule less than $5 \mathrm{~cm}$ in diameter or 2-3 nodules less than or equal to 3 $\mathrm{cm}$ in diameter) but that has not met the indication for resection. Local ablation or transarterial chemoembolization is recommended during the waiting period for LT. However, LT for HCC beyond the Milan criteria is uncertainly suggested..$^{10}$ In addition, in Korea, living donor LT is actively performed as an alternative to deceased donor liver transplantation because of the insufficient number of donors. The survival rate of living donor LT does not differ from that of deceased donor LT, but the disease-free survival rate is worse after living donor $\mathrm{LT}^{10,11}$

This single-center retrospective analysis aimed to investigate the recurrence rate of HCC after transplantation and the patterns of recurrence in LT patients compared to non-LT patients and to determine prognostic factors based on explant pathology.

\section{MATERIALS AND METHODS}

\section{Patient selection and clinicopathologic parameters}

We divided the study participants into two groups: the LT group and a control group (non-LT group). The LT group included 338 patients who had been pathologically diagnosed with HCC based on LT specimens and who had available medical records and formalin-fixed paraffin blocks of tumor tissue from the archives of the Department of Pathology of Seoul National University Hospital (SNUH) from 1996 to 2012. The non-LT group comprised 520 patients who were pathologically diagnosed with HCC based on partial hepatectomy specimens and who had available medical records and formalin-fixed paraffin blocks of tumor tissue from the archives of the Department of Pathology of SNUH from 1995 to 2006. Patients with combined hepatocellular carcinoma and cholangiocarcinoma or intrahepatic cholangiocarcinoma were excluded. The clinical information collected from existing medical records was age, sex, surgical method, underlying etiology of liver disease, serum $\alpha$-fetoprotein (AFP), preoperative treatment, and postoperative tumor recurrence. Pathological information was tumor size, number of tumors, site of tumors in the liver, gross tumor type, Edmondson-Steiner (ES) nuclear grade for HCCs, cellular type of tumor cells, histo- logical pattern, vascular invasion, pathological American Joint Committee on Cancer (AJCC) stage, whether the cancer met criteria such as the Milan criteria or UCSF criteria based on pathology, and positivity of CK19 staining, all of which were collected from pathology reports or slide reviews. The AJCC staging followed the liver tumor staging guidelines of the American Joint Committee on Cancer, seventh edition. ${ }^{12}$ The clinicopathologic parameters followed "General rules for the study of primary liver cancer of Japan." ${ }^{13}$ This study was approved by the Institutional Review Board of SNUH (H-1011-046-339). The demographic details of all patients are summarized in Table 1 according to treatment method.

\section{Immunohistochemistry of CK19}

All diagnoses were confirmed by examination of $3-\mu \mathrm{m}$ hematoxylin and eosin-stained sections of representative formalin-fixed paraffin-embedded blocks. The slides were automatically stained using a Bond-III Automated IHC/ISH stainer and a Bond Polymer Refine Detection Kit (Leica Microsystems $\mathrm{GmbH}$, Wetzlar, Germany). Positive staining with CK19 antibody (mouse monoclonal anti-human cytokeratin 19, clone RCK108, Cat. M0888, 1:200, Dako, Carpinteria, CA, USA) was observed in the cytoplasm and cellular membrane. The criterion for positivity was moderate or strong intensity in $\geq 5 \%$ of tumor cells. CK19 positivity occurred in 243 cases in the LT group and 519 cases in the non-LT group.

\section{Survey of disease progress and classification of recurrence pattern}

DFS was defined as the time to local or distant progression. Progression was diagnosed when patients experienced symptoms due to a mass lesion, when serum AFP level increased, or when computed tomography, magnetic resonance imaging, or positron emission tomography reported a new lesion or increasing tumor size. Pathologic confirmation was not necessary. The term intrahepatic progress was used when the tumor recurrence was located only in the liver, and "extrahepatic progress" was used when a metastatic tumor was identified beyond the liver with or without hepatic recurrence. The follow-up period was 36 months (median; range, 0 to 202 months) for the LT group and 73 months (median; range, 0 to 213 months) for the non-LT group.

\section{Statistical analysis}

Comparative analyses of discontinuous variables were conducted using the chi-square $\left(\chi^{2}\right)$ test, Fisher exact test, or Monte-Carlo resampling methods for small samples. Survival curves were 
Table 1. Demography of patients in LT and non-LT groups

\begin{tabular}{|c|c|c|c|}
\hline & $\mathrm{LT}(\mathrm{n}=338)$ & Non-LT $(n=520)$ & $p$-value \\
\hline Sex & & & $.039^{b}$ \\
\hline Male & $266(79)$ & $438(84)$ & \\
\hline Female & $72(21)$ & $82(16)$ & \\
\hline Age (yr) & & & .094 \\
\hline$\leq 55$ & $192(57)$ & $265(51)$ & \\
\hline$>55$ & $146(43)$ & $255(49)$ & \\
\hline Size (cm) & & & $<.001^{b}$ \\
\hline$\leq 5$ & $298(88)$ & $312(60)$ & \\
\hline$>5$ & $40(12)$ & $208(40)$ & \\
\hline Multiplicity & & & $<.001^{b}$ \\
\hline Single & $151(45)$ & $392(75)$ & \\
\hline Multiple & $187(55)$ & $128(25)$ & \\
\hline Gross type & & & $<.001^{b}$ \\
\hline Expanding nodular, etc. & $239(71)$ & $345(66)$ & \\
\hline Multinodular confluent & $73(22)$ & $174(33)$ & \\
\hline Diffuse/Infiltrative & $26(8)$ & $1(0)$ & \\
\hline Extent & & & $<.001^{b}$ \\
\hline Confined liver & $288(85)$ & $394(76)$ & \\
\hline Invasion of capsule & $45(13)$ & $87(17)$ & \\
\hline Extension to other organ & $5(1)$ & $39(8)$ & \\
\hline Angioinvasion & & & $<.001^{\mathrm{b}}$ \\
\hline Absent & $269(80)$ & $275(53)$ & \\
\hline Present & $69(20)$ & $245(47)$ & \\
\hline Large vessel invasion & & & .347 \\
\hline Absent & $324(96)$ & $491(94)$ & \\
\hline Present & $14(4)$ & $29(6)$ & \\
\hline ES nuclear grade & & & $.002^{b}$ \\
\hline Grade 1-2 & $251(74)$ & $334(64)$ & \\
\hline Grade 3-4 & $87(26)$ & $186(36)$ & \\
\hline Cell type & & & $<.001^{\mathrm{b}}$ \\
\hline Hepatic & $337(100)$ & $475(91)$ & \\
\hline Non-hepatic & $1(0)$ & $45(9)$ & \\
\hline Histologic pattern & & & $<.001^{b}$ \\
\hline Trabecular & $333(99)$ & $429(83)$ & \\
\hline Non-trabecular & $5(1)$ & $91(18)$ & \\
\hline Expression of CK19 & & & .348 \\
\hline Negative & $223(66)$ & $479(92)$ & \\
\hline Positive & $24(7)$ & $40(8)$ & \\
\hline Preoperative treatment ${ }^{c}$ & & & $<.001^{b}$ \\
\hline Done & $190(56)$ & $228(44)$ & \\
\hline Not done & $107(32)$ & $285(55)$ & \\
\hline Resection margin & & & $.003^{b}$ \\
\hline RO & $335(99)$ & $482(93)$ & \\
\hline $\mathrm{R} 1 / 2$ & $3(1)$ & $38(7)$ & \\
\hline Underlying CLD & & & $.023^{b}$ \\
\hline Viral & $290(86)$ & $460(88)$ & \\
\hline Non-viral & $48(14)$ & $53(10)$ & \\
\hline Preoperative AFP $(\mathrm{ng} / \mathrm{mL})^{c}$ & & & $.016^{b}$ \\
\hline$\leq 20$ & $191(57)$ & $250(48)$ & \\
\hline$>20$ & $144(43)$ & $270(52)$ & \\
\hline AJCC stage & & & $<.001^{\mathrm{b}}$ \\
\hline Stage I-II & $300(89)$ & $410(79)$ & \\
\hline Stage III-IV & $38(11)$ & $110(21)$ & \\
\hline
\end{tabular}

\begin{tabular}{lccc}
\hline & $\mathrm{LT}(\mathrm{n}=338)$ & Non-LT $(\mathrm{n}=520)$ & $\mathrm{p}$-value \\
\hline Milan criteria & & & $<.001^{\mathrm{b}}$ \\
$\quad$ Within & $193(57)$ & $170(33)$ & \\
$\quad$ Beyond & $145(43)$ & $350(67)$ & \\
UCSF criteria & & & $<.001^{\mathrm{b}}$ \\
$\quad$ Within & $253(75)$ & $331(64)$ & \\
$\quad$ Beyond & $85(25)$ & $189(36)$ & \\
Follow up, median & $36(0-202)$ & $73(0-213)<.001^{\mathrm{b}}$ \\
(range, mo) & & &
\end{tabular}

Values are presented as number (\%).

LT, liver transplantation; Expanding nodular, etc. including vaguely nodular, expanding nodular with or without perinodal extension; ES nuclear grade, Edmonson Steiner nuclear grade; CK19, cytokeratin 19; CLD, chronic liver disease; AFP, $\alpha$-fetoprotein; AJCC, American Joint Committee on Cancer; UCSF, University of California at San Francisco.

${ }^{a} p$-value of chi-square test, or Fisher exact test; ${ }^{b} p<.05$; ' $S$ Some data were not available: 48 preoperative treatment, 7 etiology of CLD, and 3 preoperative AFP data.

calculated using Kaplan-Meier analysis and compared by logrank testing. Multivariate analysis was performed using a Cox proportional hazards model. Multivariate analysis employing Cox regression analysis was conducted. The results were considered statistically significant when p-values were $<0.05$. Most of the tests were performed using IBM SPSS ver. 22 (IBM Corp., Armonk, NY, USA), and the Monte-Carlo resampling method was performed by "coin" package of $\mathrm{R}$ statistics. ${ }^{14-16}$

\section{RESULTS}

\section{Clinicopathologic characteristics and prognostic parameters of LT and non-LT}

As compared in Table 1, many clinicopathologic characteristics of patients in the LT and non-LT groups were different. The LT group had a higher proportion of males, single lesions, expanding nodular gross type, confined lesions in the liver, hepatic histologic type, trabecular pattern, and underlying viral chronic liver disease (CLD) than in the non-LT group (all $\mathrm{p}<.05$ ). Tumor size was smaller, angioinvasion was less frequent, and ES-nuclear grade and preoperative AFP were lower in the LT group than in the non-LT (all p <.05). Age, CK19-positive expression rate, and large vessel invasion were not different between the two groups. The proportion of patients in a low AJCC stage (I-II stage) and the proportion of those within Milan or UCSF criteria were higher in the LT than in the non-LT group (Table 1). In summary, patients in the LT group had more differentiated tumor histology and a lower stage of HCC than patients in the non-LT group. Hazard ratios of DFS according to the clinicopathologic parameters, which were calculated by univariate Cox proportional hazard model, are plotted in Fig. 1. The prognostic factors associated 
with DFS were similar between the LT and non-LT groups. Size, multiplicity of tumor, extent of tumor, angioinvasion, large vessel invasion, ES nuclear grade, positive expression of CK19, resection margin, and preoperative AFP were significant prognostic factors of DFS in both the LT and non-LT groups (all $\mathrm{p}<.05)$. Gross type was a prognostic factor in the LT group but not in the non-LT group, and this was probably due to the rarity of diffuse/infiltrative type in the non-LT group. Sex, age, tumor cell type, histologic pattern, underlying causes of CLD, and preoperative treatment were not prognostic factors in either the LT or non-LT group. Lower AJCC stage and adherence to the Milan criteria and UCSF criteria were associated with a better DFS (all $\mathrm{p}<.05$ ), but the hazard ratios of higher AJCC stage and non-adherence to the criteria compared to lower AJCC stage and adherence to the criteria were higher in the LT group than in the non-LT group $(7.328,8.727,5.451$ vs 2.312, 1.849, 1.719 , respectively, all $\mathrm{p}<.05)$.

\section{Multivariable analysis of DFS rate of resected HCCs}

Significant prognostic factors in univariate analysis in both the LT and non-LT groups were analyzed with transplantation or non-transplantation by multivariable analysis with the Cox proportional hazards model in AJCC stage I-II patients (Table 2). Surgical method was an independent prognostic factor, as were extent of tumor, multiplicity of tumor, angioinvasion, preoperative AFP, size of tumor, and ES nuclear grade. CK19

HR, disease-free survival

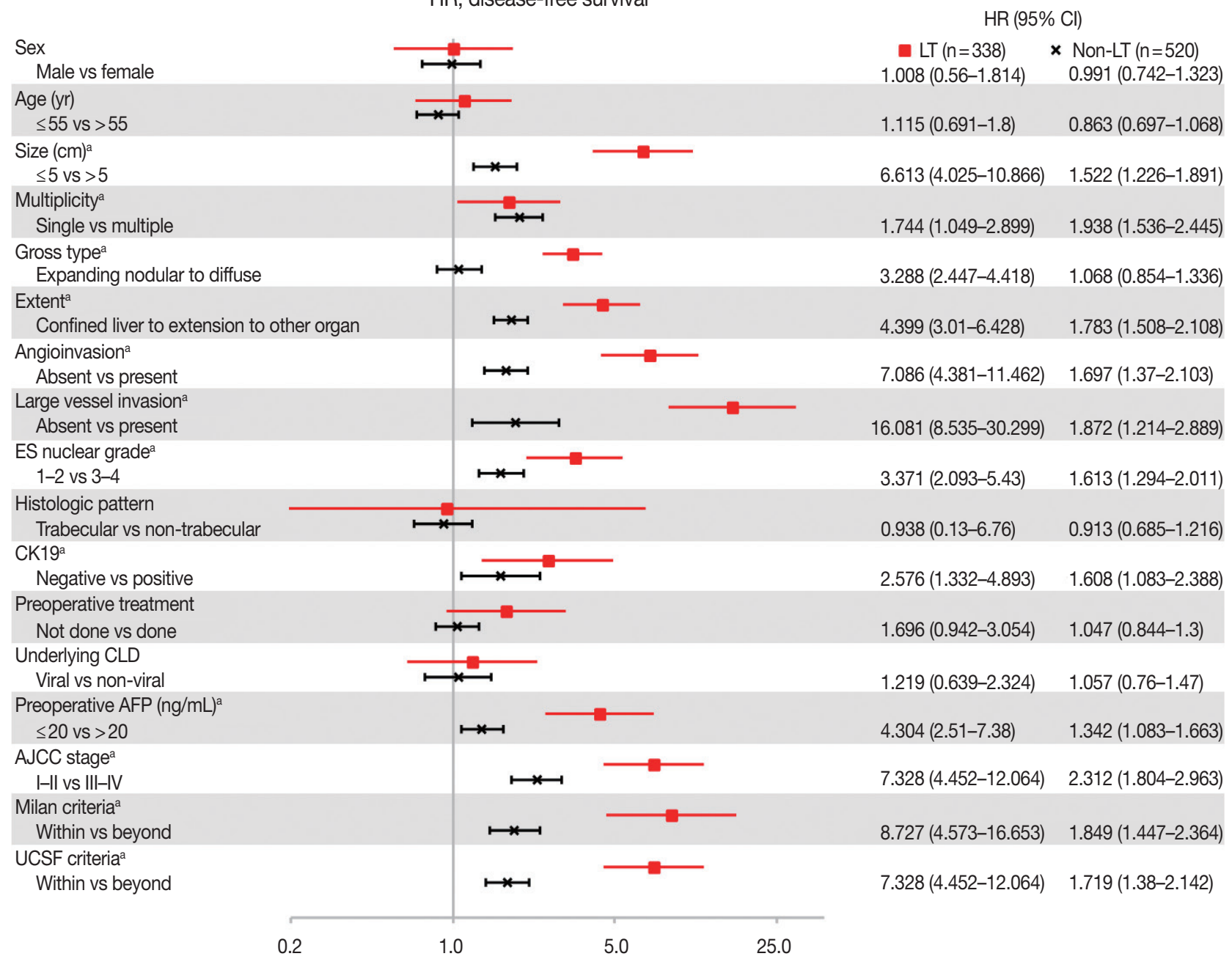

Fig. 1. Comparison of prognostic factors between the liver transplantation (LT) group and the non-LT group by univariate disease-free survival analysis. HRs of LT and non-LT groups calculated by Cox proportional hazard model are plotted in each row. HR (95\% CI), hazard ratio (95\% confidence interval) by Cox proportional hazard model; LT, liver transplantation; ES nuclear grade, Edmonson Steiner nuclear grade; CLD, chronic liver disease; AFP, $\alpha$-fetoprotein; AJCC, American Joint Committee on Cancer; UCSF, University of California at San Francisco criteria. ${ }^{2}$ Parameters are statistically significant prognostic factors (log-rank $\left.\mathrm{p}<.05\right)$. 
expression and gross type were not independent prognostic factors. The hazard ratio of LT was 0.321 (95\% confidence interval, 0.235 to 0.439 ) compared to non-LT, showing that it might have a protective effect against HCC progress.

\section{Disease-free survival patterns of the LT and non-LT groups}

Cumulative DFS is plotted in Fig. 1 with DFS rates in each year according to the AJCC stage (Fig. 2). In the total group, patients who underwent LT had a better DFS than non-LT patients (5-year DFS rates, $79 \%$ vs $35 \%$, respectively in LT and non-LT, log-rank $\mathrm{p}<.001)$. Further analysis was performed according to AJCC stage, with the entire patient group divided into two groups based on stage I-II and stage III-IV classification. LT in patients with stage I-II HCC resulted in better DFS compared to the non-LT group (5-year DFS rates, $84 \%$ vs $40 \%$, respectively; log-rank $\mathrm{p}<.001)$. No difference was observed in DFS between the LT and non-LT groups for stage III-IV HCC (5-year DFS rates, $23 \%$ vs $15 \%$; log-rank $\mathrm{p}=.295)$ (Fig. 2). As shown in Fig. 1A and B, recurrence in the LT group occurred in the early period after surgery, and cumulative DFS was sustained at approximately $80 \%$ after 3 years.

\section{Tumor recurrence site and time in the LT and non-LT groups according to stage}

The recurrence site and post-operative period of recurrence were compared according to stage and resection method (Table 3). Among patients with tumors classified as stage I-II HCC, 43 patients in the LT group and 250 in the non-LT group experi-

Table 2. Multivariable analysis of disease-free survival rate of stage $\mathrm{I}-\mathrm{II}$ hepatocellular carcinomas $(\mathrm{n}=710)$

\begin{tabular}{llllc}
\hline & Beta & SE & p-value & Hazard ratio (95\% Cl) \\
\hline Extent of tumor & 0.476 & 0.083 & $<.001^{\mathrm{a}}$ & $1.61(1.367-1.896)$ \\
Operation (non-LT vs LT) & -1.135 & 0.159 & $<.001^{\mathrm{a}}$ & $0.321(0.235-0.439)$ \\
Multiplicity (single vs multiple) & 0.365 & 0.115 & $.001^{\mathrm{a}}$ & $1.44(1.15-1.803)$ \\
Angioinvasion (absent vs present) & 0.33 & 0.116 & $.004^{\mathrm{a}}$ & $1.391(1.108-1.746)$ \\
Preoperative AFP ( $\leq 20 \mathrm{ng} / \mathrm{mL}$ vs $>20 \mathrm{ng} / \mathrm{mL})$ & 0.266 & 0.106 & $.012^{\mathrm{a}}$ & $1.305(1.061-1.606)$ \\
Size of tumor ( $\leq 5 \mathrm{~cm}$ vs $>5 \mathrm{~cm}$ ) & 0.256 & 0.115 & $.026^{\mathrm{a}}$ & $1.291(1.032-1.617)$ \\
ES nuclear grade (grade 1-2 vs 3-4) & 0.235 & 0.111 & $.034^{\mathrm{a}}$ & $1.265(1.017-1.573)$ \\
CK19 (negative vs positive) & 0.3 & 0.178 & .093 & $1.35(0.952-1.916)$ \\
Gross type & 0.165 & 0.101 & .102 & $1.18(0.968-1.438)$
\end{tabular}

SE, standard error; Cl, confidence interval; LT, liver transplantation; AFP, a-fetoprotein; ES nuclear grade, Edmonson-Steiner nuclear grade; CK19, cytokeratin 19. ${ }^{\mathrm{a}} \mathrm{p}<.05$.

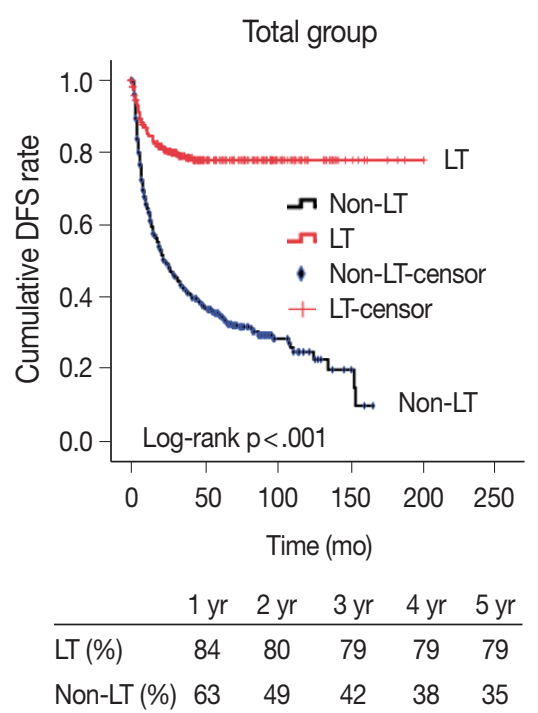

AJCC stage I-II

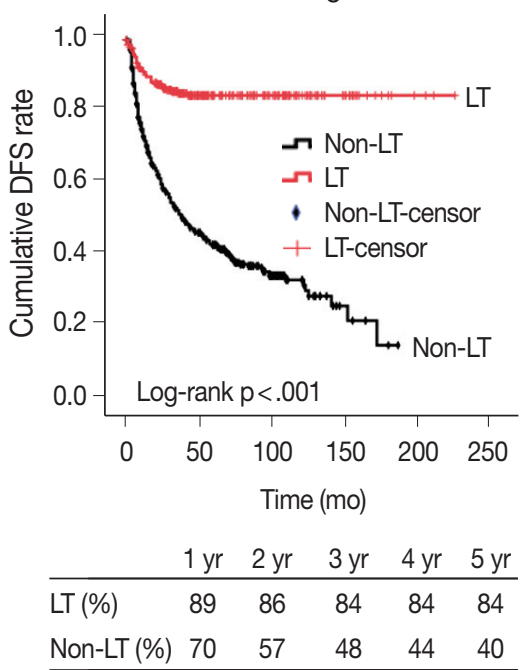

AJCC stage III-IV

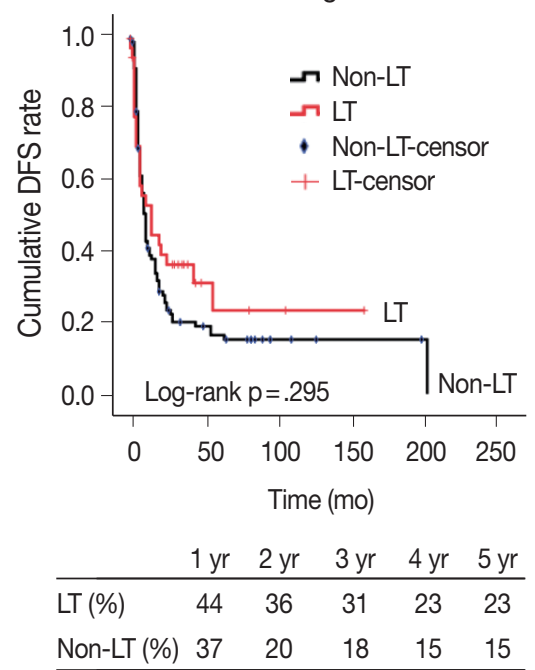

(A)

B

Fig. 2. Kaplan-Meier curves for disease-free survival (DFS) between the liver transplantation (LT) group and the non-LT group. (A) The DFS of patients with any stage disease was significantly longer in the LT group than in the non-LT group $(p<.001)$. (B) Among patients with stage I- II disease, DFS was also longer in the LT group than in the non-LT group $(p<.001)$. (C) There were no significant differences in the DFS of stage III-IV patients between the LT and non-LT groups. AJCC, American Joint Committee on Cancer. 
Table 3. Comparison of recurrence pattern between LT and non-LT

\begin{tabular}{|c|c|c|c|c|c|c|}
\hline & \multirow{2}{*}{ No. } & \multicolumn{2}{|c|}{ Intrahepatic } & \multicolumn{2}{|c|}{ Extrahepatic } & \multirow{2}{*}{$p$-value } \\
\hline & & $\leq 2 \mathrm{yr}$ & $>2 \mathrm{yr}$ & $\leq 2 \mathrm{yr}$ & $>2 \mathrm{yr}$ & \\
\hline \multicolumn{7}{|c|}{ AJCC stage I-II } \\
\hline \multirow[t]{2}{*}{ LT } & 43 & \multicolumn{2}{|c|}{$9(21)$} & \multicolumn{2}{|c|}{$34(79)$} & $<.001^{c}$ \\
\hline & & $9(100)$ & 0 & $30(88)$ & $4(12)$ & .376 \\
\hline \multirow[t]{2}{*}{ Non-LT } & 250 & \multicolumn{2}{|c|}{$155(62)$} & \multicolumn{2}{|c|}{95 (38) } & \\
\hline & & $29(57)$ & $66(43)$ & $79(83)$ & $16(17)$ & $<.001^{\mathrm{c}}$ \\
\hline \multicolumn{7}{|c|}{ AJCC stage III-IV } \\
\hline \multirow[t]{2}{*}{ LT } & 25 & \multicolumn{2}{|c|}{$9(36)$} & \multicolumn{2}{|c|}{$16(64)$} & .198 \\
\hline & & $8(89)$ & $1(11)$ & $15(94)$ & $1(6)$ & .511 \\
\hline \multirow[t]{2}{*}{ Non-LT } & 87 & \multicolumn{2}{|c|}{$44(51)$} & \multicolumn{2}{|c|}{$43(49)$} & \\
\hline & & $41(93)$ & $3(9)$ & $41(95)$ & $2(5)$ & .600 \\
\hline
\end{tabular}

LT, liver transplantation; AJCC, American Joint Committee on Cancer.

${ }^{a}$ Intrahepatic recur vs extrahepatic recur; ${ }^{b} \leq 2$ yr vs $>2$ yr; ${ }^{c} p<.05$.

enced tumor progress. Within the LT group, there were nine cases $(21 \%)$ with intrahepatic progress and 34 (79\%) with extrahepatic progress. In contrast, the non-LT group included 155 cases (62\%) of intrahepatic recurrence and 95 cases $(38 \%)$ of extrahepatic progress. Metastasis to extrahepatic organs was the predominant pattern of recurrence in the LT group among patients with stage I-II HCC, whereas intrahepatic recurrence was predominant in the non-LT group $(\mathrm{p}<.001)$. This pattern disappeared in patients with stage III-IV HCC. There were nine cases $(36 \%)$ of intrahepatic progress and 16 cases $(64 \%)$ of extrahepatic progress within the LT group, and the non-LT group included 44 cases (51\%) of intrahepatic recurrence and 43 cases (49\%) of extrahepatic progress, but there was no significant difference between the two groups $(\mathrm{p}=.198)$. As previously mentioned, the time to recurrence and recurrence site were different between the LT and non-LT groups. All nine intrahepatic recurrence cases of AJCC stage I-II LT patients recurred within 2 years, but 66 of 155 intrahepatic recurrence cases $(43 \%)$ of AJCC stage I-II non-LT patients occurred beyond 2 years $(\mathrm{p}<.001)$. Most of the extrahepatic metastasis occurred within 2 years in both the LT and non-LT groups for AJCC stage I-II stage patients (30 [88\%] vs 79 [83\%], respectively, $\mathrm{p}=.376$ ). This progress pattern also disappeared in stage III-IV patients who showed early progress within 2 years after surgery, regardless of recurrence sites (Table 3).

\section{DISCUSSION}

Our study analyzed the recurrence rate of HCC and the recurrence pattern in LT patients compared with non-LT patients, as well as prognostic factors after transplantation. Recurrence after LT usually occurred within 2 years and was more frequently identified in extrahepatic sites; in contrast, recurrence after non-LT frequently occurred beyond 2 years after surgery, and the sites of recurrence were intrahepatic. Furthermore, this difference was clear in stage I-II HCC, but was not observed in stage III-IV HCC.

Many previous studies comparing LT and other treatment choices for HCC reported that patients who underwent LT had a better survival than those who underwent resection. ${ }^{17}$ The rationale of LT as a treatment choice for HCC is loco-regional removal of tumor and protective removal of the damaged liver, which plays a major role in the de novo recurrence of HCC. The results of our study support this dual rationale for LT to treat HCC. In our study, intrahepatic recurrence was more frequent in the non-LT group than in the LT group in patients with stage I-II and was sustained for a long period after surgery. Therefore, it is thought that LT is an effective treatment to suppress de novo carcinogenesis, which is the main mechanism of late recurrence of $\mathrm{HCC}^{18}$

In the present study, the high proportion of extrahepatic HCC recurrence after LT was similar to results from other studies. ${ }^{19}$ Because of the protective removal of the source of de novo carcinogenesis in LT, tumor recurrence in LT patients can be mainly explained by metastasis or regrowth of remnant tumor cells. Assuming complete resection (R0 resection), potential tumor cells do not exist in the liver after LT. Circulating tumor cells (CTCs) might be considered to explain this phenomenon. According to a recent study, primary tumor cell invasion to the bloodstream and CTCs are responsible for tumor recurrence and metastasis, including that of HCC. ${ }^{20-22}$

Although LT is the treatment of choice for early-stage HCC, it is not a feasible choice because of the shortage of deceased donors. Increasing living donor LT has compensated for the shortage of 
organ donations, but maintaining a balance between the benefit to the recipient and the risk to the living donor is another emerging issue in this field. Patient selection criteria tend to expand the range of recipients by up regulation of number or size of tumor. This classical approach used to be based on preoperative radiologic studies. However, the histology and biologic features of the tumor are important prognostic factors for predicting tumor progress. ${ }^{23}$ Similar to other studies, the multivariate analysis of prognostic factors in our study also concluded that ES nuclear grade was an independent prognostic factor. However, although CK19 expression was not an independent prognostic factor in our study, it was reported as a prognostic marker of HCC recurrence beyond the Milan criteria in another study. ${ }^{9}$

Another issue concerning the histologic factors of HCC in LT is the gap between preoperative clinical staging and post-operative pathologic staging. The Milan criteria and UCSF criteria consider vascular invasion as an important exclusion criterion. Because the degree of vasculature that can be examined by radiologic study and pathologic study is much different, many cases within the criteria before surgery are changed to beyond the criteria after surgery based on the pathology of the explant. In our study, the Milan and UCSF criteria were applied by explant pathology. Pretransplant tumor staging and explant pathology do not always correlate, and 83 of 145 cases $(57 \%)$ within the Milan criteria were classified as "beyond Milan" based on the presence of angioinvasion in microscopic findings. There might be discrepancies in the size or number of tumors between the pre-transplant radiological staging and explant pathologic staging. ${ }^{24,25}$ Therefore, further studies to reduce the preoperative and postoperative staging are needed.

In conclusion, this study demonstrated that DFS was better in the LT group compared to the non-LT group for patients with stage I-II HCC. Accordingly, there might be a tendency for tumors to occur in the late period after surgery in patients who have not undergone LT. Extrahepatic recurrence is more frequent than intrahepatic recurrence after LT. Once tumor recurrence has occurred, dissemination to additional locations occurs easily. The prognostic factors in patients who have undergone LT are similar to those observed in previous studies; LT, extent of tumor, multiplicity of tumor, angioinvasion, preoperative AFP, size of tumor, and ES nuclear grade were independent prognostic factors.

\section{Conflicts of Interest}

No potential conflict of interest relevant to this article was reported.

\section{Acknowledgments}

This research was supported by a grant of the Korea Health Technology R\&D Project through the Korea Health Industry Development Institute (KHIDI), funded by the Ministry of Health \& Welfare, Republic of Korea (grant number: HI14C3298).

\section{REFERENCES}

1. Parkin DM, Bray F, Ferlay J, Pisani P. Estimating the world cancer burden: Globocan 2000. Int J Cancer 2001; 94: 153-6.

2. Mazzaferro V, Regalia E, Doci R, et al. Liver transplantation for the treatment of small hepatocellular carcinomas in patients with cirrhosis. N Engl J Med 1996; 334: 693-9.

3. Tan KC, Rela M, Ryder SD, et al. Experience of orthotopic liver transplantation and hepatic resection for hepatocellular carcinoma of less than $8 \mathrm{~cm}$ in patients with cirrhosis. Br J Surg 1995; 82: 253-6.

4. McPeake JR, O'Grady JG, Zaman S, et al. Liver transplantation for primary hepatocellular carcinoma: tumor size and number determine outcome. J Hepatol 1993; 18: 226-34.

5. Yao FY, Ferrell L, Bass NM, et al. Liver transplantation for hepatocellular carcinoma: expansion of the tumor size limits does not adversely impact survival. Hepatology 2001; 33: 1394-403.

6. Gondolesi GE, Roayaie S, Muñoz L, et al. Adult living donor liver transplantation for patients with hepatocellular carcinoma: extending UNOS priority criteria. Ann Surg 2004; 239: 142-9.

7. Todo S, Furukawa H; Japanese Study Group on Organ Transplantation. Living donor liver transplantation for adult patients with hepatocellular carcinoma: experience in Japan. Ann Surg 2004; 240: 451-9.

8. Lee SG, Hwang S, Moon DB, et al. Expanded indication criteria of living donor liver transplantation for hepatocellular carcinoma at one large-volume center. Liver Transpl 2008; 14: 935-45.

9. Miltiadous O, Sia D, Hoshida Y, et al. Progenitor cell markers predict outcome of patients with hepatocellular carcinoma beyond Milan criteria undergoing liver transplantation. J Hepatol 2015; 63: 1368-77.

10. Korean Liver Cancer Study Group (KLCSG); National Cancer Center, Korea (NCC). 2014 KLCSG-NCC Korea Practice Guideline for the Management of Hepatocellular Carcinoma. Gut Liver 2015; 9: 267-317.

11. Hwang S, Lee SG, Joh JW, Suh KS, Kim DG. Liver transplantation for adult patients with hepatocellular carcinoma in Korea: comparison between cadaveric donor and living donor liver transplanta- 
tions. Liver Transpl 2005; 11: 1265-72.

12. Edge SB, Compton CC. The American Joint Committee on Cancer: the 7th edition of the AJCC cancer staging manual and the future of TNM. Ann Surg Oncol 2010; 17: 1471-4.

13. The general rules for the clinical and pathological study of primary liver cancer. Liver Cancer Study Group of Japan. Jpn J Surg 1989; 19: 98-129.

14. R Core Team. R: A language and environment for statistical computing [Internet]. Vienna: R Foundation for Statistical Computing, 2015 [cited 2016 Nov 1]. Available from: https://www.R-project. org/.

15. Hothorn T, Hornik K, van de Wiel MA, Zeileis A. Implementing a class of permutation tests: the coin package. J Stat Softw 2008; 28: 1-23.

16. Hothorn T, Hornik K, van de Wiel MA, Zeileis A. A Lego system for conditional inference. Am Stat 2006; 60: 257-63.

17. Kaido T, Morita S, Tanaka S, et al. Long-term outcomes of hepatic resection versus living donor liver transplantation for hepatocellular carcinoma: a propensity score-matching study. Dis Markers 2015; 2015: 425926

18. Imamura H, Matsuyama $Y$, Tanaka E, et al. Risk factors contributing to early and late phase intrahepatic recurrence of hepatocellular carcinoma after hepatectomy. J Hepatol 2003; 38: 200-7.
19. Scortegagna E, Jr., Karam AR, Sioshansi S, Bozorgzadeh A, Barry C, Hussain S. Hepatocellular carcinoma recurrence pattern following liver transplantation and a suggested surveillance algorithm. Clin Imaging 2016; 40: 1131-4.

20. Attard G, de Bono JS. Utilizing circulating tumor cells: challenges and pitfalls. Curr Opin Genet Dev 2011; 21: 50-8.

21. Zhang Y, Li J, Cao L, Xu W, Yin Z. Circulating tumor cells in hepatocellular carcinoma: detection techniques, clinical implications, and future perspectives. Semin Oncol 2012; 39: 449-60.

22. Zhang Y, Shi ZL, Yang X, Yin ZF. Targeting of circulating hepatocellular carcinoma cells to prevent postoperative recurrence and metastasis. World J Gastroenterol 2014; 20: 142-7.

23. Donat M, Alonso S, Pereira F, et al. Impact of histological factors of hepatocellular carcinoma on the outcome of liver transplantation. Transplant Proc 2016; 48: 1968-77.

24. Mejia GA, Gomez MA, Serrano J, et al. Correlation between the radiologic and histologic size of hepatocellular carcinoma in patients eligible for liver transplantation. Transplant Proc 2006; 38: 1394-5.

25. El Moghazy W, Kashkoush S, Meeberg G, Kneteman N. Incidence, characteristics, and prognosis of incidentally discovered hepatocellular carcinoma after liver transplantation. J Transplant 2016; 2016: 1916387. 